Research

\title{
The exclusive induction of extinction is gated by BDNF
}

\author{
Anne Kirtley ${ }^{1}$ and Kerrie L. Thomas ${ }^{1,2}$ \\ ${ }^{1}$ Cardiff School of Biosciences, Cardiff University, Cardiff CF10 3AX, United Kingdom
}

\begin{abstract}
We have previously reported that the reconsolidation and extinction of hippocampal-dependent contextual fear memory can be initiated by a single context conditioned stimulus (CS) presentation of either short or long duration, and that both processes require protein synthesis in this brain region. Furthermore, reconsolidation depends on Zif268 activity in this region. Here we show that by infusing a recombinant brain-derived neurotrophic factor ( $r$ BDNF) directly into the brain of rats, that high levels of mature BDNF in the hippocampus at retrieval constrain the extinction of the fear memory after prolonged memory recall. We also show after a short CS exposure that reconsolidation was impaired using antisense oligonucleotides targeting Zif268, and that, similarly, reductions in conditioned behavior were observed after prolonged CS presentation when extinction is constrained by high levels of BDNF. This is direct evidence that in the mammalian brain extinction proceeds exclusively after prolonged CS exposure. In addition, that BDNF activity in the hippocampus contributes to a molecular switch for the extinction of hippocampal-dependent memory.
\end{abstract}

[Supplemental material is available online at http://www.learnmem.org.]

Memory extinction is characterized by the weakening of the conditioned behavioral response (CR) that occurs when a conditioned stimulus (CS) is presented without reinforcement of a biologically salient unconditioned stimulus (US). Extinction is currently considered as new learning in which the CS predicts the absence, rather than the occurrence, of the CS (formation of a CS-no US association [Rescorla 2001]). The extinction memory subsequently competes with the original association (CS-US) for control of behavior. However, the cued recall of a memory can also cause it to become labile through a process of destabilization, rendering it susceptible to interference, and the original memory requires restabilization for long-term retention (Nader et al. 2000). This process is called reconsolidation.

The exact conditions of memory retrieval determine whether reconsolidation and extinction are engaged. For example, exposure to CS for a short duration triggers reconsolidation, whilst extinction is engaged following exposure for more extended, or repeated, CS presentations (Eisenberg et al. 2003; Pedreira and Maldonado 2003; Suzuki et al. 2004). These earlier studies did not however distinguish whether the two processes were mutually exclusive or, alternatively, were triggered in parallel but that one process dominated. Studies in Chasmagnathus have shown that the offset of the CS is the signal that triggers either reconsolidation or extinction (Pedreira et al. 2004). Following contextual fear conditioning (CFC), a hippocampal-dependent form of longterm memory, we have shown that protein synthesis inhibition in the dorsal hippocampus disrupts reconsolidation after a single short exposure to the context CS and extinction following prolonged CS exposure (Lee et al. 2004; Barnes and Thomas 2008). This is consistent with earlier reports of the effects of systemic and regional administration of protein synthesis on the two postretrieval memory processes in a range of aversive memory tasks (Nader et al. 2000; Debiec et al. 2002; Kida et al. 2002; Eisenberg et al. 2003; Pedreira and Maldonado 2003; Suzuki et al. 2004), but see (Lattal and Abel 2001, 2004). Our results with CFC showing that protein synthesis inhibitors infused into the hippocampus

\footnotetext{
${ }^{2}$ Corresponding author.
}

E-mail ThomasKL5@cf.ac.uk.

Article is online at http://www.learnmem.org/cgi/doi/10.1101//m.1877010. can impair both reconsolidation and extinction after the recall of contextual fear memory (CFM) suggest that reconsolidation and extinction processes may not only proceed independently, but there may be a molecular switch in the hippocampus that controls the expression of mutually exclusive memory processes associated with CFM. Indeed, recent evidence based on the activation of CREB, a gene regulated during memory processing (Hall et al. 2001b), in the hippocampus after different durations of conditioned context exposure demonstrates that the differential regulation of plasticity-associated genes was correlated with reconsolidation and extinction (Mamiya et al. 2009). However, it was not shown whether CREB activation in the hippocampus was causal for each process, a response to either, or the result of an interaction between them.

To investigate the characteristics of a molecular switch in the hippocampus, we investigated the hippocampal expression of two memory-associated molecules, brain-derived neurotrophic factor (BDNF) and Zif268, which we have shown to be selectively associated with extinction and reconsolidation, respectively. We also observed the effects of manipulating their levels during recall on the subsequent expression of CFM. Here we show that by infusing recombinant BDNF protein (rBDNF) directly into the hippocampus, high levels of BDNF were refractory for extinction produced by a single prolonged exposure to the CS. Antisense oligonucleotide targeting Zif268 mRNA for inactivation (Zif268ASO) blocked reconsolidation, but failed to produce stable amnesia after prolonged exposure to the CS when extinction was constrained. Nevertheless, inhibiting Zif268 activity unmasked extinction in the short term. This shows that, similar to Chasmagnathus, extinction proceeded exclusively of reconsolidation in the mammalian brain. Moreover, the combined activities of BDNF and Zif268 switch off extinction. However, although we also show here that increased expression of BDNF and Zif268 were measured in CA1 after reconsolidation-inducing short CS exposures, we have previously shown that reducing hippocampal BDNF levels with BDNFASO prior to short recall (which should be predicted to permit extinction) has no effect on the reactive CR (Lee et al. 2004). Therefore, while BDNF may act as a switch for extinction after prolonged CS exposures, it may not be similarly controlling extinction after short recall. Thus, the activities of BDNF and Zif268 in the hippocampus together do not adequately describe the 


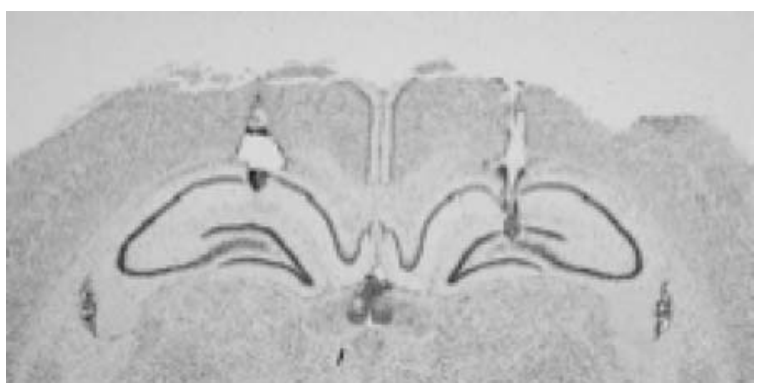

Figure 1. Representative photomicrograph showing the bilateral infusion site in dorsal CA1.

molecular switch for reconsolidation and extinction during a single CS presentation.

\section{Results}

Sixty rats were used in this study. Forty-eight rats underwent surgery for behavioral analysis. Histological analysis showed that the cannulae terminated bilaterally in the dorsal hippocampus in all of the operated rats and none showed signs of gross damage to the hippocampus (Fig. 1). One operated rat was excluded from the study and analysis because of a blocked cannula during the training infusion.

\section{Experiment 1: Exogenous rBDNF impairs the extinction of CFC}

After CFC, prolonged (10 $\mathrm{min}$ ) but not short (2 min) re-exposure to the training context led to a decrease in conditioned fear behavior measured during a long-term memory retrieval test $2 \mathrm{~d}$ later (LTM1) (Fig. 2). The extinction of the fear memory was persistent and was maintained for at least $23 \mathrm{~d}$. In contrast, repeated short context exposures during extinction training $3 \mathrm{~h}, 2 \mathrm{~d}$, and $21 \mathrm{~d}$ later did not lead to any appreciable change in the behavioral expression of the CFM.

We have previously shown that attenuating the cleavage of endogenous proBDNF to mBDNF in the dorsal hippocampus during extinction training promotes extinction (Barnes and Thomas 2008). This suggested that the ratio of precursor to mature forms of BDNF might be a key determinant for extinction of CFM. To further test this idea, we examined the effects of directly increasing hippocampal mBDNF levels during extinction training on CFM. Infusing rBDNF into the dorsal hippocampus prior to a prolonged 10-min re-exposure to the conditioned context rapidly impaired the extinction of the fear memory and this effect on LTM was sustained. This manifested as no difference in the conditioned responding to the context between the rats not undergoing extinction (Short recall group) and those undergoing extinction in the presence of rBDNF (rBDNF group) during the PR-STM trial $3 \mathrm{~h}$ after extinction training, or at any of the LTM tests $2-23 \mathrm{~d}$ later. Therefore, it appears that hippocampal mBDNF opposes the extinction of CFM by constraining acquisition. There was no effect of rBDNF on freezing behavior during the extinction training session (within-session extinction).

\section{Experiment 2: Exogenous rBDNF reveals a delayed expression CFM in the presence of Zif268ASO}

Infusing Zif268ASO into the hippocampus prior to a short exposure to the conditioned context resulted in the long-lasting decrease in fear memory (Fig. 3). This observation was interpreted as an effect of the Zif268ASO on the reconsolidation of LTM
(Lee et al. 2004). It is not known whether Zif268 plays a role in extinction. After administering Zif268ASO into the dorsal hippocampus, we showed that a prolonged 10-min exposure to the conditioned context resulted in a reduction of conditioned freezing behavior measured at the PR-STM test and long-term retrieval tests, LTM1 and LTM 2, compared to the behavior during the first $2 \mathrm{~min}$ of extinction training $\left(F_{(1.826,7.306)}=5.264, P=\right.$ $0.040, \varepsilon=0.609$, repeated measures ANOVA). Similarly, infusions of the missense sequence, Zif268MSO, had no effect on withinsession and between-session extinctions (Supplemental Fig. S1). A planned comparison between rats administered Zif268ASO and Zif268MSO before extinction training indicated that the subsequent behavioral responses in both groups were similar. (Test $x$ Infusion interaction $F_{(3.831,34.547)}=1.603, P=0.198, \varepsilon=0.547$, repeated measures ANOVA). Thus, extinction of CFM was not affected by intrahippocampal infusions of Zif268ASO. However, the coadministration of rBDNF and Zif268ASO before extinction training had a marked effect on conditioned freezing measured in the LTM tests, manifesting as a recovery of the conditioned behavior between the first LTM1 test, $2 \mathrm{~d}$ after extinction training and the second LTM 2 test $21 \mathrm{~d}$ later.

\section{Experiment 3: Zif268 and BDNF IX expression are} differentially regulated by short and long recalls of CFM We observed that the length of exposure to the conditioned context during extinction training resulted in distinct transcriptional events in CA1 (Fig. 4). Thirty min after a brief 2-min exposure to
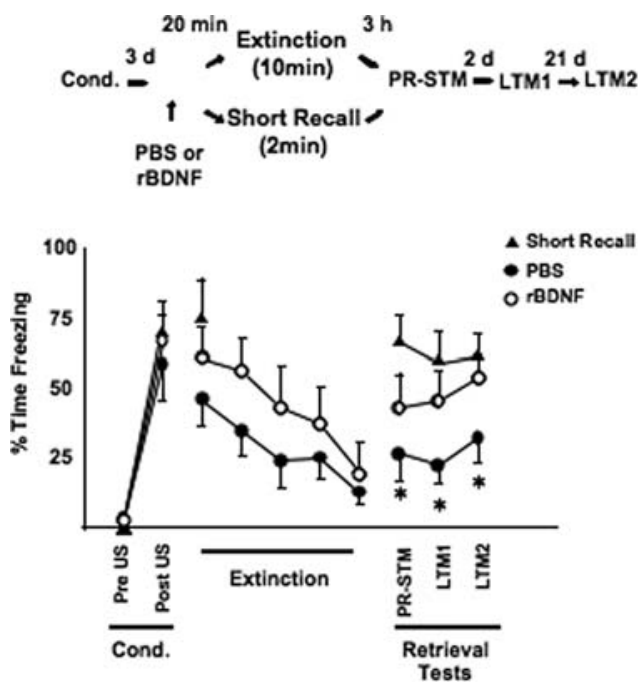

Figure 2. Intrahippocampal infusion of rBDNF attenuates the longterm decrease in conditioned freezing behavior initiated by a prolonged exposure to the conditioning context. Rats were exposed to the conditioning context (CS) during either a 2-min Short Recall $(n=5)$ or a 10-min Extinction $(n=6)$ training trial following infusions of PBS, or for a 10-min trial after rBDNF $(n=7)$. All rats showed an increase in freezing behavior in the post-US compared to pre-US conditioning period (main effect of Test, $F_{(1,15)}=96.753, P=0.000$, repeated measures ANOVA). A 10-min exposure to the CS resulted in a within-session reduction of conditioned freezing behavior during extinction training $\left(F_{(2.373,26.101)}=\right.$ $6.965, P=0.000, \varepsilon=0.593$, repeated measures ANOVA), but there was no effect of the rBDNF on the within-session fear responses (Test $x$ Infusion interaction, $F_{(2.373,26.101)}=0.081, P=0.593, \varepsilon=0.754$, repeated measures ANOVA). Prolonged exposure to the CS resulted in a reduction in conditioned freezing responses in the PBS $(P=0.034$, Tukey's test), but not the rBDNF group $(P=0.227$, Tukey's test) during the subsequent retrieval tests. This was sustained for at least $21 \mathrm{~d}$ after extinction training ( ${ }^{*} P<0.05$ PBS compared to Short Recall, Tukey's test). 


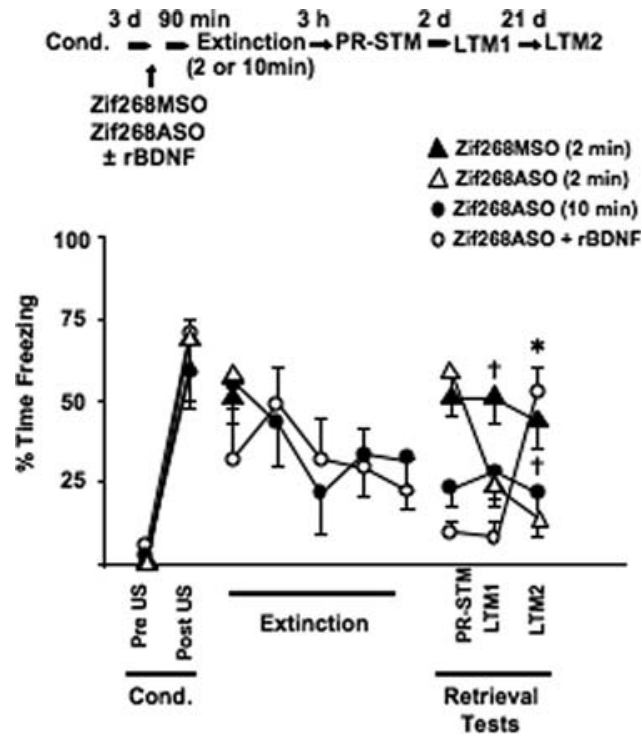

Figure 3. Coinfusion of rBDNF and Zif268ASO prior to extinction training by a single 10-min exposure to the context recovers conditioned freezing behavior $21 \mathrm{~d}$ later. Rats received a single conditioning trial. Ninety min prior to extinction, $3 \mathrm{~d}$ later, they either received Zif268MSO $(n=6)$, Zif268ASO $(n=6$ [2-min recall] or $n=5$ [10-min recall]), or Zif268ASO and $\operatorname{rBDNF}(n=6)$. Zif268ASO infusion prior to a short 2-min CS exposure produced a loss of the CR during the retrieval tests compared to the Zif268MSO administered rats (Test $\times$ Infusion interaction, $F_{(1.362,13.624)}=8.704, P=0.007, \varepsilon=0.681$, repeated measures ANOVA), which was characterized by a reduction in freezing behavior at LTM1 and LTM2. Zif268ASO alone or Zif268ASO coinfused with rBDNF had no effect on the decrement in the fear response measured during the 10-min extinction training (Extinction $\times$ Infusion interaction, $F_{(3.021,27.185)}=1.086, P=0.378, \varepsilon=0.755$, repeated measures ANOVA). There were no differences in the rats' behavior during conditioning prior to intrahippocampal infusions (Conditioning $\times$ Infusion interaction, $F_{(3,19)}=0.185, P=0.905$, repeated measures ANOVA). However, there was an effect of coinfusing rBDNF with Zif268ASO compared to Zif268ASO only on conditioned freezing behavior during LTM1 and LTM2 retrieval tests $\left(F_{(1.718,15.459)}=17.885, P=0.000, \varepsilon=\right.$ 0.859 , repeated measures ANOVA). Results are presented as the mean \pm SEM. ${ }^{\dagger} p<0.05$ Zif268ASO (2 min) vs. Zif268MSO (2 min). ${ }^{*} P<0.05$ Zif268ASO + rBDNF compared to Zif268ASO groups.

the conditioned context there was an up-regulation in the mRNA levels of BDNF IX and Zif268 compared to the no recall control rats that were fear conditioned but not re-exposed to the context. The increase in BDNF IX expression was specific to the CA1, and was not seen in CA3 or in the dentate gyrus (data not shown). In contrast, only Zif268 expression was increased in CA1 after a prolonged 10-min exposure that results in extinction of the CFM.

\section{Discussion}

Here we show that a prolonged, but not a short exposure, of fearconditioned rats to the conditioned context produces extinction of CFM that was maintained for at least $3 \mathrm{wk}$. Prolonged exposure to the context CS was accompanied by an increase in Zif268 expression in the CA1 of conditioned animals, whereas brief re-exposure correlates with the induction of both Zif268 and $B D N F I X$ expression. The infusion of rBDNF protein into the hippocampus before prolonged context re-exposure impaired extinction. More specifically, we showed that high levels of mBDNF functioned to prevent the acquisition of the CS-no US extinction memory. Intrahippocampal infusion of Zif268ASO, which we have previously reported to prevent the reconsolidation of CFM after recall (Lee et al. 2004), had no apparent effect on extinction. However, coinfusion of rBDNF with Zif268ASO into the hippocampus prior to extinction training resulted in the recovery, or unmasking, of CFM 3 wk later. This demonstrates that the original association was intact after extinction, and that extinction but not reconsolidation mechanisms were engaged by a single prolonged exposure to the CS. It also indicates that under conditions of retrieval that favor the extinction of LTM, Zif268 may not be acting to reconsolidate the active recalled memory, but conversely plays a role in attenuating extinction.

In contrast to other studies using the CFC paradigm (e.g., Kimura et al. 2008; Archbold et al. 2010), we saw no spontaneous recovery of the behavioral performance after extinction training. Studies with fear conditioning to a discrete cue identified several factors that affect the resistance of extinction to recovery. These include the interval between conditioning and extinction (Myers et al. 2006; Woods and Bouton 2008; Huff et al. 2009; but see Alvarez et al. 2007; Schiller et al. 2008; Archbold et al. 2010), the interval between extinction and test (Bouton 1993, 2004; Quirk 2002), the level of fear behavior at extinction (Maren and Chang 2006), and the number and pattern of nonreinforced trails (Cain et al. 2003; Urcelay et al. 2009). These factors would be expected to interact with each other such that the precise experimental conditions used by independent laboratories would differentially reveal recovery of the cued fear responses. Although most accounts of extinction and spontaneous recovery suggest that the original learning remains unchanged, there is ultimately a dissipation of the extinction memory at the behavioral level, the mechanisms underlying the loss of the extinction trace or its control over behavioral responses remain to be determined (for review, see Rescorla 2004). The durable extinction we show here may be a consequence of the context entering into primary excitatory and inhibitory associations with the US during conditioning and extinction, respectively. As such, the context acts as an explicit rather than hierarchical stimulus to facilitate the retrieval of both excitatory and inhibitory associations with equal efficiency and probability, which then compete for behavioral control depending on their relative strengths. It remains to be empirically tested whether loss of the CR measured here $21 \mathrm{~d}$ after a prolonged 10-min exposure to the context reflects a permanent extinction memory, or that the precise conditions of conditioning and extinction delay or alter the rate of the recovery process. Our extinction training per se is unlikely to have produced amnesia of the original fear memory because recovery of CR can be seen when Zif268ASO and rBDNF are administered before training.

Neither the expression, nor the reduction, of the conditioned freezing behavior over the course of extinction training (within-session extinction) was affected by infusions of rBDNF into the hippocampus. This indicates that rBDNF does not affect the hippocampal-dependent processes that mediate the retrieval of CFM (Fanselow 2000), or the habituation of conditioned fear responses (Kamprath and Wotjak 2004). The impairment of extinction by rBDNF was both rapid and sustained because freezing behavior was comparable to rats that had not undergone extinction training (short recall group) at the earliest postretrieval test (PR-STM) $3 \mathrm{~h}$ later and at long-term retrieval tests for at least $21 \mathrm{~d}$. This shows that increasing mBDNF in the hippocampus around the time of a nonreinforced trial impairs the acquisition of the extinction of CFM. This is consistent with our recent study showing that decreasing the levels of mBDNF in the CA1 using tPA-STOP (to inhibit the proteolytic cleavage of proBDNF to $\mathrm{mBDNF}$ ) resulted in a potentiation of extinction (Barnes and Thomas 2008). Therefore, altering the ratio of proBDNF to mBDNF in the hippocampus, and perhaps the CA1 specifically has profound effects on the extinction of CFM. 
A

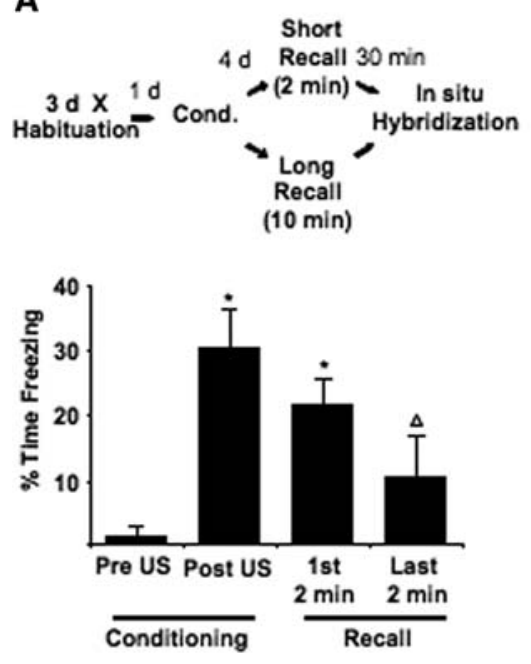

C

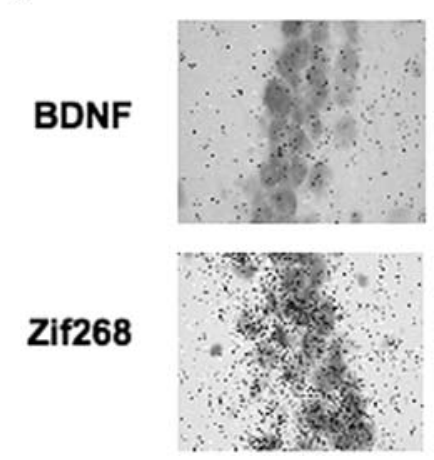

B

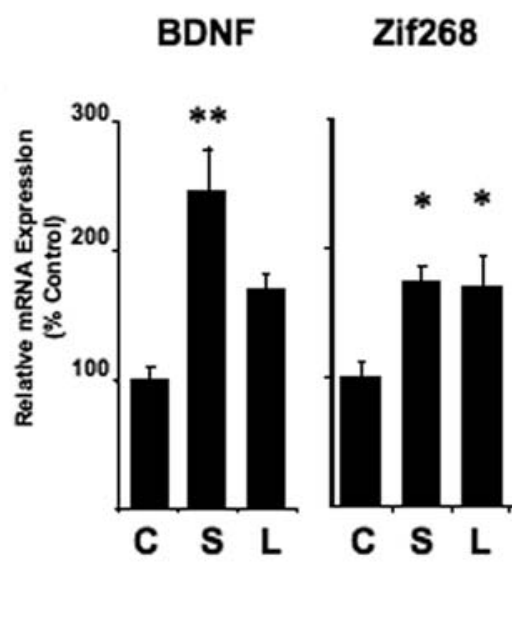

$\mathbf{S}$
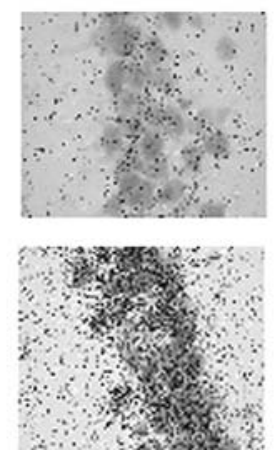

$\mathbf{L}$
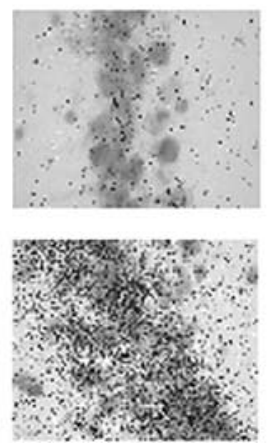

Figure 4. Reconsolidation and extinction of contextual fear memory results in the differential regulation of Bdnf and Zif268 expression in hippocampal CA1. (A) Rats $(n=12)$ were habituated to a fear conditioning apparatus for $10 \mathrm{~min} / \mathrm{d}$ for $3 \mathrm{~d}$ prior to conditioning. Recall of the fear memory $4 \mathrm{~d}$ later consisted of exposing rats to the conditioned context for either 2 min (S, short recall $n=4$ ) or 10 min (L, long recall, $n=4)$. Control rats $(C, n=4)$ did not undergo recall. Repeated measures ANOVA revealed significant main effects of the training and test phases on freezing behavior $\left(F_{(3,9)}=\right.$ $4.576, P=0.033)$. This manifested as an increase in freezing behavior post-US during the conditioning period and in the first $2 \mathrm{~min}$ of the recall test $4 \mathrm{~d}$ later. Rats exposed to the conditioned context for 10 min showed a reduction in freezing during the last 2 min on the recall test. $* P<0.05$ compared to pre-US, $\Delta P<0.05$ compared to first 2 min during recall. $(B)$ Thirty min after recall in situ hybridization revealed regulation in the expression of the coding exon IX of $B d n f\left(F_{(2,9)}=13.913, P=0.002\right.$, ANOVA) and Zif268 $\left(F_{(2,9)}=6.322, P=0.019\right)$ in the CA1 of the hippocampus. This was due to an increase in the expression of both genes after short exposure to the CS (S). In contrast, there was an increase in Zif268 but not BDNF IX mRNA levels ( $P=0.091$, Sidak's test) after a longer 10-min exposure to the CS (L). ${ }^{*} P<0.05,{ }^{* *} P<0.01$ compared to the No recall control (C, Sidak's test). Grains per CA1 neuron (mean \pm SEM) in the control No Recall group (C): Bdnf IX, $13.84 \pm 1.28$; Zif268, $179.59 \pm$ 22.59. (C) Photomicrographs (100X) of small dark silver grains associated with CA1 pyramidal cells identifying the expression of BDNF IX and Zif268 from an individual rat from the C, S, and L groups.

The cellular mechanisms that underlie these evidently opposing effects of the different BDNF species on extinction have yet to be identified. ProBDNF-mediated signaling facilitates longterm depression (LTD) in the hippocampus via the activation of the p75NTR neurotrophin receptor (Woo et al. 2005). This suggests that extinction may be associated with plasticity mechanisms similar to LTD. Indeed, there have been numerous reports of an association between LTD (or depotentiation of synapses) and the extinction of LTM since one of the first studies to support this idea (Quirk et al. 1995). Interestingly, it is becoming evident that experimental manipulations that disrupt LTD result in impairments in the acquisition of extinction (Sotres-Bayon et al.

2007; Dalton et al. 2008; Duffy et al. 2008). Therefore, it is possible that LTD mechanisms initiated by proBDNF-mediated signaling events support the acquisition of extinction.

An alternative proposition is that mBDNF, or mBDNF-mediated signaling events, play a direct role in extinction by preventing or impairing extinction. However, BDNF and TrkB signaling, the cogent receptor for $\mathrm{mBDNF}$, are required for the extinction of aversive memories (Chhatwal et al. 2006; Gruart et al. 2007; Heldt et al. 2007). It is possible that the optimal level of mBDNF plays a role in the consolidation of extinction and that deviation from this level (too much or too little) results in an impairment of long-term extinction. Recently it has been suggested that transient and sustained activation of the TrkB receptor by mBDNF have different consequences for downstream transcriptional and synaptic structural events in hippocampal neurons (Ji et al. 2010). For example, transient activations of TrkB by mBDNF were correlated with either transient or no increases in Arc expression, and increases in the density of spines. Conversely, sustained activations result in prolonged increases in Arc and little change in spine density. Evidence suggests that transient activations in TrkB signaling may initiate extinction. Consistent with this idea, compared to short recall conditions, Arc expression is constrained after prolonged exposures to the CS that lead to the extinction of CFM (Barnes and Thomas 2008; Mamiya et al. 2009; see also Guzowski et al. 2001). In addition, experimental conditions that reduce endogenous mBDNF levels in the hippocampus potentiate extinction of CFM (Barnes and Thomas 2008). Furthermore, fewer dendritic spines correlate with impaired extinction (Ryu et al. 2008). In our experiments high levels of exogenously administered rBDNF at the time of extinction training may disrupt the finely controlled mechanisms that endogenous mBDNF orchestrate to consolidate extinction.

It has been previously established that upon a single CS presentation, the competition between extinction and reconsolidation are governed by the precise conditions of memory reactivation, conditions that include the duration of the CS presentation (Eisenberg et al. 2003; Pedreira and Maldonado 2003; Suzuki et al. 2004; Barnes and Thomas 2008). Nevertheless, it is not clear whether there is a mutual exclusion between reconsolidation and extinction because either the retrieval conditions trigger only one of the processes at CS offset (Pedreira et al. 2004; Perez-Cuesta and Maldonado 2009) or there is an interaction between the two processes triggered in parallel (Mamiya et al. 2009).

To address this issue we infused both Zif268ASO and rBDNF prior to prolonged exposure to the CS. If extinction was 
exclusively triggered by long recall sessions, we predicted the preservation of conditioned fear because rBDNF would constrain extinction. If both processes were initiated under conditions when extinction was impaired, no fear response would be measured at LTM1 or LTM2, as the original memory supporting the CR would not undergo ZIf268-dependent reconsolidation (Lee et al. 2004, 2005). Thus, amnesia would ensue. We showed that following extinction training combined with coadministration of the Zif268ASO and rBDNF, no conditioned freezing was seen at the long-term retrieval test $2 \mathrm{~d}$ later. This may suggest that reconsolidation was engaged by a prolonged CS exposure, and that both extinction and reconsolidation processes are initiated by a single CS presentation. However, conditioned fear reemerged 3 wk later. The recovery of the fear memory demonstrates a fundamental feature of the process initiated by a single prolonged nonreinforced exposure to the conditioning context; that the ability of the CS to elicit the CR is not lost after prolonged CS exposure, but rather it is actively inhibited at some level (Pavlov 1927; Rescorla and Wagner 1972; Robbins 1990; Bouton 2002). Therefore, only one process, extinction, was engaged after prolonged exposure to the CS.

Our data indicate that Zif268 likely plays a role in the extinction of CFM since Zif268 expression was increased in CA1 after recall, and the disruptive effects of direct rBDNF infusions into the hippocampus on the consolidation of extinction are ameliorated by the coadministration of the Zif268ASO. Although this effect of Zif268ASO was transient, we also showed that direct Zif268ASO administration prior to short recall impaired the expression of reactive memory. This importantly demonstrates that Zif268ASO has specific and long-lasting effects on the hippocampal function. It appears that Zif268 inhibition facilitates extinction, and that Zif268-mediated events in the hippocampus may therefore protect against or act to prevent extinction at least in the short-term. However, a more recent study in which Zif268 was conditionally overexpressed in the forebrain failed to show changes in the extinction of conditioned taste aversion (CTA) (Baumgartel et al. 2008). However, the failure to observe a role for Zif268 in extinction may be due to the already high levels of Zif268 in the insular cortex, a structure integral for CTA, occluding the effect of additional protein on the behavior. Therefore, low levels of mBDNF and Zif2868 in the dorsal hippocampus at retrieval are permissive for the extinction of CFM.

Zif268 expression is up-regulated in CA1 after both short and long exposures to the context CS. Contrarily, the expression of $B D N F I X$ is specifically increased following short durations of CS exposure, similar to the refractory changes in the Arc levels in CA1 (see above), a BDNF-regulated protein (Waltereit et al. 2001; Yin et al. 2002; Ying et al. 2002), with prolonged CS exposures. The differential regulation of BDNF IX and Zif268 expression in the CA1 provides a conceptual framework for the role for the interaction of BDNF and Zif268 activities in the hippocampus in controlling the extinction of CFM, not only under conditions of recall that favor extinction, but under all situations following the retrieval of a nonreinforced CS.

It could be argued that Zif268-mediated events act to inhibit the extinction of LTM after retrieval. Phenomenologically, a blockade of reconsolidation and facilitation of extinction by Zif268ASO would be the same since both would be predicted to lead to a decrease in the CR at later LTM tests. So the question whether Zif268-mediated events play a role in both reconsolidation and extinction or, alternatively, acts to prevent extinction under all conditions of recall may reduce to determining whether reconsolidation is exclusively triggered after short recall in the mammalian brain as in the crab (Pedreira et al. 2004). High levels of endogenous BDNF after short recall may inhibit extinction in a similar manner to the experimental manipulation of hippocampal BDNF in long recall as we observe here. However, BDNFASO has no effect on CFM after short recall (Lee et al. 2004) demonstrating that the memory process(es) engaged is not sensitive to changes in hippocampal BDNF levels. This implies that extinction is not triggered after short recall. Furthermore, Zif268ASO selectively impairs the reconsolidation process engaged after short recall.

Thus, we show that a Zif268-mediated process supports reconsolidation but impairs extinction. This is analogous to the role of the transcription factor NFKB in these two processes (Merlo et al. 2005; Lubin and Sweatt 2007; Merlo and Romano 2008). However, Zif268 does not act as a direct molecular switch between reconsolidation and extinction because the administration of Zif268ASO alone before extinction training had no effect on the extent or duration of extinction. We propose that Zif268 modulates the long-term extinction dependent on mBDNF activity. As such optimal levels of endogenous mBDNF and BDNF-mediated events are required for the long-term extinction per se, but Zif268-mediated mechanisms may negatively regulate extinction at least in the short term. In analogy to the distinct molecular mechanisms that underlie the expression of memory during the consolidation of LTM (McGaugh 1966, 2000; Davis and Squire 1984; Emptage and Carew 1993; Izquierdo et al. 1998, 2002; Stough et al. 2006), this may reflect that the expression of the extinction memory may be supported by different, and potentially independent, molecular mechanisms shortly after training compared to those required for persistent extinction.

Together our data from studies in the mammalian brain support the findings in Chasmagnathus that reconsolidation and extinction mechanisms are mutually exclusive when triggered by a single CS (Merlo et al. 2005; Merlo and Romano 2008; Perez-Cuesta and Maldonado 2009). Moreover, it appears to be evolutionarily conserved that the extent of extinction training (CS exposure) determines whether extinction learning is engaged. We propose that BDNF in the hippocampus act as a molecular switch with low levels triggering extinction but high levels above a threshold are refractory for extinction. This is analogous to BDNF acting via a NOT gate for extinction. However, our data here also suggest the interaction of the activities of BDNF and Zif268 at recall in CA1 on the subsequent expression of CFM is acting via a NAND gate (no reactive CR when either or both BDNF and Zif268 levels are low, Table 1). Thus, high levels of BDNF and Zif268 and their associated activities in the hippocampus may act to switch off extinction. Indeed, the spontaneous recovery when Zif268 levels alone are low at recall (caused by the administration of the rBDNF and Zif268ASO cocktail) is indicative of a less persistent, and potentially weaker, extinction memory. Furthermore, this would indicate a correlation between low BDNF and Zif268 activities and the persistence of extinction. This hypothesis would predict spontaneous recovery after long recall since only Zif268 levels are high. Although we did not see this, this may be because a longer interval than $21 \mathrm{~d}$ between extinction and test is required to observe recovery, and/or the roles of BDNF and Zif268 are either not functionally equivalent or equally impacted by the doses of rBDNF and Zif268 ASO we used in this study. It will be interesting to explore whether extinction that undergoes spontaneous recovery in other experimental situations can be made more resistant to recovery by inhibiting Zif268 activity during extinction training. Our results also show that Zif268-mediated processes act to support reconsolidation. Thus Zif268 may always function to maintain or preserve the original CS-US association. However, we previously reported that intrahippocampal BDNFASO prior to short recall conditions resulted in CR (reconsolidation) at later LTM tests (Lee et al. 2004). These are conditions under which Zif268 levels are high and BDNF levels would be experimentally lowered and would be 
Table 1. Two input logic gate truth table for reactive CFM

\begin{tabular}{|c|c|c|c|c|c|}
\hline Experimental condition & BDNF & Zif268 & $\begin{array}{c}\text { No CR/ } \\
\text { extinction } \\
\text { (impaired } \\
\text { reconsolidation) }\end{array}$ & $\begin{array}{l}\text { Spontaneous } \\
\text { recovery }\end{array}$ & Notes \\
\hline Long recall + Zif268ASO & 0 & 0 & Yes & No & Persistent extinction \\
\hline $\begin{array}{l}\text { Long recall } \\
\text { Short recall + BDNFASO }\end{array}$ & $\begin{array}{l}0 \\
0\end{array}$ & $\begin{array}{l}1 \\
1\end{array}$ & $\begin{array}{l}\text { Yes } \\
\text { No }\end{array}$ & No & $\begin{array}{l}\text { Persistent extinction } \\
\text { Reconsolidation }\end{array}$ \\
\hline $\begin{array}{l}\text { Long recall }+ \text { rBDNF }+ \\
\text { Zif268ASO }\end{array}$ & 1 & 0 & Yes & Yes & Transient extinction \\
\hline Short recall + Zif268ASO & 1 & 0 & Yes & - & No reconsolidation \\
\hline $\begin{array}{l}\text { Long recall + rBDNF } \\
\text { Short recall }\end{array}$ & $\begin{array}{l}1 \\
1\end{array}$ & $\begin{array}{l}1 \\
1\end{array}$ & $\begin{array}{l}\text { No } \\
\text { No }\end{array}$ & - & $\begin{array}{l}\text { No extinction } \\
\text { Reconsolidation }\end{array}$ \\
\hline
\end{tabular}

Although mBDNF is required for the extinction of fear memory, high levels of hippocampal BDNF at recall appears to negatively regulate the expression of the conditioned freezing (CR) during a subsequent exposure to the nonreinforced context CS. Thus, BDNF may be acting as a NOT gate for extinction. The results of the experiments presented in this study show that the interaction of the activities of BDNF and Zif268 in the hippocampus at recall on the expression of extinction is acting via a NAND gate (no reactive CR when either or both BDNF and Zif268 levels are low). Thus, high levels of BDNF function may turn off extinction, and when Zif268 is high also, reconsolidation proceeds to supporting the CR. However, we have previously shown that under conditions of short recall (italics) and BDNFASO, CR is measured at later LTM tests (Lee et al. 2004). This suggests that the activities of BDNF and Zif268 in the hippocampus do not completely describe the switch between extinction and reconsolidation under all conditions of recall. $1=$ high BDNF or high Zif268 levels in CA1.

predicted to result in a reduction of fear-associated behavior if these two molecules acted as a simple NAND gate for extinction. This indicates that although extinction and reconsolidation of CFM are mutually exclusive processes, the switch between them is not described by the interaction of the activities of BDNF and Zif268 in the hippocampus alone. This indicates that the full description of a molecular switch between the two post-retrieval memory processes may include the activity of one or more molecules in addition to BDNF and Zif268 in the hippocampus.

\section{Materials and Methods}

\section{Subjects}

The subjects were adult male Lister hooded rats weighing 280$350 \mathrm{~g}$. They were housed in pairs, in holding rooms maintained at $21^{\circ} \mathrm{C}$ on a reversed-light cycle (12-h light/dark; lights on at 10:00 p.m.). All experiments were conducted in the dark period of the rat's day. Food and water were freely available throughout the experiment. All procedures were conducted in accordance with local Cardiff University Ethical Committee approval and the United Kingdom 1986 Animals (Scientific Procedures) Act (Project license PPL 30/2236).

\section{Surgery and microinfusions into the dorsal hippocampus} Steel double guide cannulae aimed at the dorsal hippocampus (AP -3.50 , relative to bregma) were surgically implanted under anesthesia, as described by Barnes and Thomas (2008), at least $1 \mathrm{wk}$ prior to behavioral training and the infusion of antisense oligodeoxynucleotide targeting Zif268 (Zif268ASO), missense oligodeoxynucleotide Zif268 (Zif268MSO), or recombinant human BDNF (rBDNF). Bilateral infusions via the chronically indwelling cannula were carried out in awake rats using a syringe pump, connected to injectors (28 gauge, projecting $1 \mathrm{~mm}$ beyond the guide cannulae) by polyethylene tubing. Zif268ASO was a PAGE-purified phosphorothioate end-capped 18-mer sequence complementary to nucleotides $348-365$ of M18416.1 (5'GGTAGTTGTCCATGGTGG-3', SigmaGenosys), resuspended in sterile PBS to a concentration of $2 \mathrm{nmol} / \mu \mathrm{L}$. The sequence was subjected to a BLAST search on the National Center for Biotechnology
Information BLAST server using the GenBank database. The antisense sequence had positive matches only for the target mRNA sequences, and no other rat or human coding sequences. Control Zif268MSO sequence (5'-GTGTTCGGTAGGGTGTCA-3'), which included the same 18 nucleotides as the Zif268ASO but in a scrambled order, did not generate any full matches to identified gene sequences in the database. rBDNF was the $27 \mathrm{kDa}$ dimer of two identical mature BDNF subunits (B-250, Alomone Laboratories) resuspended in sterile PBS to a concentration of $0.25 \mu \mathrm{g} / \mu \mathrm{L}$. One or $2 \mathrm{~d}$ prior to behavioral training, awake rats were infused with PBS to habituate them to the administration procedure. The volumes of all infusions were $1 \mu \mathrm{L} /$ hemisphere, and were given over $2 \mathrm{~min}$ (PBS), $8 \mathrm{~min}$ (Zif268ASO and Zif268MSO), and 15 min (rBDNF).

\section{Contextual fear conditioning}

Each rat received a conditioning trial in one of two different contexts. These contexts differed in a number of features including size, spatial location, odor, and lighting. During the 3-min conditioning training trial, rats received a single scrambled footshock $(0.5 \mathrm{~mA}$ for $2 \mathrm{sec}) 2 \mathrm{~min}$ after being placed into the conditioning context. All rats returned to the home cages after conditioning. Extinction training 3 or $4 \mathrm{~d}$ later consisted of exposing rats to the conditioned context for either 2 min or 10 min. Retrieval tests: $3 \mathrm{~h}$ (post-retrieval short-term memory, PR-STM), $2 \mathrm{~d}$ (LTM1), and $23 \mathrm{~d}$ (LTM2) after conditioning, each rat was exposed to the conditioning context for 2 min. For in situ hybridization experiments, a separate cohort of rats was conditioned, underwent extinction training, and was killed 30 min later, with the exception of one group that underwent conditioning and was killed 4 d later (No Recall group).

\section{Behavioral analysis and statistics}

Freezing behavior served as a measure of conditioned fear to the context during the conditioning, extinction training and retrieval tests of the behavioral procedures. This was video-recorded and quantified by an observer blind to the experimental group. One unit of freezing was defined as a continuous absence of movement other than that required for respiration in $1 \mathrm{sec}$ sampled every 10 sec. Data are presented as the mean \pm SEM percentage time spent freezing. Freezing behavior was analyzed in a repeated measures analysis of variance (repeated measures ANOVA) with Test as a within-subjects factor and Infusion as a between-subjects factor. For repeated measures ANOVA, Mauchly's Test of Sphericity was applied. If the sphericity assumption was not met, the Greenhouse-Geisser correction was applied. Planned comparisons were made using repeated measures ANOVA between pre-US and post-US phases of conditioning, during extinction training, and during retrieval testing (PR-STM, LTM1, and LTM2). Tukey's test was then used for post hoc analysis to determine the sources of significance and the $P$-value constrained by the number of comparisons made $(P<0.05, P<0.01$, and $P<0.001)$.

\section{Tissue collection}

Rats were killed by $\mathrm{CO}_{2}$ exposure and decapitation 30 min after behavioral testing, and whole brains were rapidly removed and frozen on dry ice. The brains were stored at $-70^{\circ} \mathrm{C}$ until in situ hybridization or histological determination of the cannulae placement using thionin staining of Nissl substance. 


\section{In situ hybridization}

Sections $(14 \mu \mathrm{m})$ were cut at $-20^{\circ} \mathrm{C}$ on a freezing microtome (Leica) and thaw-mounted onto poly-L-lysine (hydrobromide; molecular mass 300,000; Sigma, Poole)-coated glass slides (0.02 $\mathrm{mg} / \mathrm{mL}$ diethyl pyrocarbonate-treated water). The sections were air-dried for not less than $30 \mathrm{~min}$, fixed in 4\% paraformaldehyde in $0.1 \mathrm{M}$ PBS, pH 7.4, for $5 \mathrm{~min}$, rinsed in PBS for $1 \mathrm{~min}$, delipidated in $70 \%$ ethanol for $4 \mathrm{~min}$, and stored in $95 \%$ ethanol at $4^{\circ} \mathrm{C}$.

Hybridizations were performed essentially as described by Hall et al. (2001a). cDNA antisense probes (45-mers) were synthesized commercially (SigmaGenosys) complementary to nucleotides 3-47 of Zif268 (NM 012551, 5'-CCGTTGCTCAGCAGCATCATCTCCTCCAGTTTGGGGTAGTTGTCC) and nucleotides 855-899 of protein-encoding BDNF exon IX (Liu et al. 2005; Aid et al. 2007) (NM 012513.3, 5'-CGAAC-CTTCTGGTCCTCATCCAGCAGCTCTTCGA-TCACGTGCTCA). These oligonucleotides were $3^{\prime}$-end labeled with $\left[\alpha^{35} \mathrm{~S}\right]$ dATP $(1200 \mathrm{Ci} /$ mmol; Perkin Elmer-NEN) in a 30:1 molar ratio of radiolabeled ATP:oligonucleotide using terminal deoxynucleotidyl transferase (Promega) as described previously (Wisden and Morris 1994). Specific activity of the ${ }^{35}$ S-labeled probe was between $2.0 \times 10^{5}$ and $3.0 \times 10^{5} \mathrm{dpm} / \mu \mathrm{L}$ probe. To define nonspecific hybridization, adjacent slide-mounted sections were incubated with radiolabeled oligonucleotide in the presence of an excess (100X) concentration of unlabeled oligonucleotide probe. After hybridization, sections were exposed to Kodak BioMax MR X-ray film (Sigma-Aldrich Company Ltd.) for 1-2 wk. After obtaining appropriate X-ray film exposures, sections were dipped in Ilford K5 photographic emulsion (Agar Scientific). Sections were exposed for $5-10$ wk at $4{ }^{\circ} \mathrm{C}$, before development and counterstaining with $0.01 \%$ thionin.

Images were captured using LeicaQWIN imaging software (Leica Microsystems) using an 100X objective under oil immersion. Silver grain density was assessed in dorsal hippocampal CA1 pyramidal cells (approximately bregma $-3.3-3.9 \mathrm{~mm}$ ) using ImageJ imaging software (http://rsbweb.nih.gov/ij/). Grains (total and nonspecific) were counted over sufficient randomly selected neurons from each region for each animal such that the SE of the counts for any region was $<10 \%$ of the population mean (typically 24 cells). In each case cells were selected from at least three nonadjacent separate sections. A specific grain count was then calculated for each region by subtracting total and nonspecific counts. The mean silver grain count in each region for each animal was then divided by the mean count in that region for the No Recall control group to give a standardized grain count (percent) for each group. Standardized results were analyzed by ANOVA, and post-hoc comparisons were made using Sidak's test (corrected pair-wise comparisons).

\section{Acknowledgments}

We acknowledge our grant sponsor, BBSRC, UK, Grant Number, BB/C000583X/1. A.K. held a MRC Quota PhD studentship.

\section{References}

Aid T, Kazantseva A, Piirsoo M, Palm K, Timmusk T. 2007. Mouse and rat BDNF gene structure and expression revisited. J Neurosci Res 85: $525-535$.

Alvarez RP, Johnson L, Grillon C. 2007. Contextual-specificity of short-delay extinction in humans: Renewal of fear-potentiated startle in a virtual environment. Learn Mem 14: 247-253.

Archbold GE, Bouton ME, Nader K. 2010. Evidence for the persistence of contextual fear memories following immediate extinction. Eur J Neurosci 31: 1303-1311.

Barnes P, Thomas KL. 2008. Proteolysis of proBDNF is a key regulator in the formation of memory. PLOS ONE 3: e3248. doi: 10.1371/ journal.pone.0003248.

Baumgartel K, Genoux D, Welzl H, Tweedie-Cullen RY, Koshibu K, Livingstone-Zatchej M, Mamie C, Mansuy IM. 2008. Control of the establishment of aversive memory by calcineurin and Zif268. Nat Neurosci 11: 572-578.

Bouton ME. 1993. Context, time, and memory retrieval in the interference paradigms of Pavlovian learning. Psychol Bull 114: 80-99.
Bouton ME. 2002. Context, ambiguity, and unlearning: Sources of relapse after behavioral extinction. Biol Psychiatry 52: 976-986.

Bouton ME. 2004. Context and behavioral processes in extinction. Learn Mem 11: 485-494.

Cain CK, Blouin AM, Barad M. 2003. Temporally massed CS presentations generate more fear extinction than spaced presentations. Can J Exp Psychol 29: 323-333.

Chhatwal JP, Stanek-Rattiner L, Davis M, Ressler KJ. 2006. Amygdala BDNF signaling is required for consolidation but not encoding of extinction. Nat Neurosci 9: 870-872.

Dalton GL, Wang YT, Floresco SB, Phillips AG. 2008. Disruption of AMPA receptor endocytosis impairs the extinction, but not acquisition of learned fear. Neuropsychopharmacology 33: 2416-2426.

Davis HP, Squire LR. 1984. Protein synthesis and memory: A review. Psychol Bull 96: 518-559.

Debiec J, LeDoux JE, Nader K. 2002. Cellular and systems reconsolidation in the hippocampus. Neuron 36: 527-538.

Duffy S, Labrie V, Roder JC. 2008. D-serine augments NMDA-NR2B receptor-dependent hippocampal long-term depression and spatial reversal learning. Neuropsychopharmacology 33: 1004-1018.

Eisenberg M, Kobilo T, Berman DE, Dudai Y. 2003. Stability of retrieved memory: Inverse correlation with trace dominance. Science $\mathbf{3 0 1}$ : 1102-1104.

Emptage NJ, Carew TJ. 1993. Long-term synaptic facilitation in the absence of short-term facilitation in Aplysia neurons. Science 262: 253-256.

Fanselow MS. 2000. Contextual fear, gestalt memories, and the hippocampus. Behav Brain Res 110: $73-81$.

Gruart A, Sciarretta C, Valenzuela-Harrington M, Delgado-Garcia JM, Minichiello L. 2007. Mutation at the TrkB PLC $\gamma$-docking site affects hippocampal LTP and associative learning in conscious mice. Learn Mem 14: 54-62.

Guzowski JF, Setlow B, Wagner EK, McGaugh JL. 2001. Experience-dependent gene expression in the rat hippocampus after spatial learning: A comparison of the immediate-early genes Arc, c-fos, and zif268. J Neurosci 21: 5089-5098.

Hall J, Thomas KL, Everitt BJ. 2001a. Cellular imaging of zif268 expression in the hippocampus and amygdala during contextual and cued fear memory retrieval: Selective activation of hippocampal CA1 neurons during the recall of contextual memories. J Neurosci 21: 2186-2193.

Hall J, Thomas KL, Everitt BJ. 2001b. Fear memory retrieval induces CREB phosphorylation and Fos expression within the amygdala. Suppl Eur J Neurosci 13: 1453-1458.

Heldt SA, Stanek L, Chhatwal JP, Ressler KJ. 2007. Hippocampus-specific deletion of BDNF in adult mice impairs spatial memory and extinction of aversive memories. Mol Psychiatry 12: 656-670.

Huff NC, Hernandez JA, Blanding NQ, LaBar KS. 2009. Delayed extinction attenuates conditioned fear renewal and spontaneous recovery in humans. Behav Neurosci 123: 834-843.

Izquierdo I, Izquierdo LA, Barros DM, Mello e Souza T, de Souza MM, Quevedo J, Rodrigues C, Sant'Anna MK, Madruga M, Medina JH. 1998. Differential involvement of cortical receptor mechanisms in working, short-term and long-term memory. Behav Pharmacol 9: 421-427.

Izquierdo LA, Barros DM, Vianna MR, Coitinho A, deDavid e Silva T, Choi H, Moletta B, Medina JH, Izquierdo I. 2002. Molecular pharmacological dissection of short- and long-term memory. Cell Mol Neurobiol 22: 269-287.

Ji Y, Lu Y, Yang F, Shen W, Tang TT, Feng L, Duan S, Lu B. 2010. Acute and gradual increases in BDNF concentration elicit distinct signaling and functions in neurons. Nat Neurosci 13: 302-309.

Kamprath K, Wotjak CT. 2004. Nonassociative learning processes determine expression and extinction of conditioned fear in mice. Learn Mem 11: 770-786.

Kida S, Josselyn SA, de Ortiz SP, Kogan JH, Chevere I, Masushige S, Silva AJ. 2002. CREB required for the stability of new and reactivated fear memories. Nat Neurosci 5: 348-355.

Kimura R, Silva AJ, Ohno M. 2008. Autophosphorylation of $\alpha$ CaMKII is differentially involved in new learning and unlearning mechanisms of memory extinction. Learn Mem 15: 837-843.

Lattal KM, Abel T. 2001. Different requirements for protein synthesis in acquisition and extinction of spatial preferences and context-evoked fear. J Neurosci 21: 5773-5780.

Lattal KM, Abel T. 2004. Behavioral impairments caused by injections of the protein synthesis inhibitor anisomycin after contextual retrieval reverse with time. Proc Natl Acad Sci 101: 4667-4672.

Lee JL, Everitt BJ, Thomas KL. 2004. Independent cellular processes for hippocampal memory consolidation and reconsolidation. Science 304: 839-843.

Lee JL, Di Ciano P, Thomas KL, Everitt BJ. 2005. Disrupting reconsolidation of drug memories reduces cocaine-seeking behavior. Neuron 47: 795-801. 
Liu QR, Walther D, Drgon T, Polesskaya O, Lesnick TG, Strain KJ, de Andrade M, Bower JH, Maraganore DM, Uhl GR. 2005. Human brain derived neurotrophic factor (BDNF) genes, splicing patterns, and assessments of associations with substance abuse and Parkinson's disease. Am J Med Genet B Neuropsychiatr Genet 134B: 93-103.

Lubin FD, Sweatt JD. 2007. The IkB kinase regulates chromatin structure during reconsolidation of conditioned fear memories. Neuron 55: 942-957.

Mamiya N, Fukushima H, Suzuki A, Matsuyama Z, Homma S, Frankland PW, Kida S. 2009. Brain region-specific gene expression activation required for reconsolidation and extinction of contextual fear memory. J Neurosci 29: 402-413.

Maren S, Chang CH. 2006. Recent fear is resistant to extinction. Proc Natl Acad Sci 103: $18020-18025$.

McGaugh JL. 1966. Time-dependent processes in memory storage. Science 153: $1351-1358$.

McGaugh JL. 2000. Memory-a century of consolidation. Science 287: $248-251$.

Merlo E, Romano A. 2008. Memory extinction entails the inhibition of the transcription factor NF-кB. PLOS ONE 3: e3687. doi: 10.1371/ journal.pone.0003687.

Merlo E, Freudenthal R, Maldonado H, Romano A. 2005. Activation of the transcription factor NF- $\mathrm{\kappa B}$ by retrieval is required for long-term memory reconsolidation. Learn Mem 12: 23-29.

Myers KM, Ressler KJ, Davis M. 2006. Different mechanisms of fear extinction dependent on length of time since fear acquisition. Learn Mem 13: 216-223.

Nader K, Schafe GE, Le Doux JE. 2000. Fear memories require protein synthesis in the amygdala for reconsolidation after retrieval. Nature 406: $722-726$.

Pavlov IP. 1927. Conditioned reflexes: An investigation of the physiological activity of the cerebral cortex. Oxford University Press, London, UK.

Pedreira ME, Maldonado H. 2003. Protein synthesis subserves reconsolidation or extinction depending on reminder duration. Neuron 38: $863-869$.

Pedreira ME, Perez-Cuesta LM, Maldonado H. 2004. Mismatch between what is expected and what actually occurs triggers memory reconsolidation or extinction. Learn Mem 11: 579-585.

Perez-Cuesta LM, Maldonado H. 2009. Memory reconsolidation and extinction in the crab: Mutual exclusion or coexistence? Learn Mem 16: $714-721$.

Quirk GJ. 2002. Memory for extinction of conditioned fear is long-lasting and persists following spontaneous recovery. Learn Mem 9: 402-407.

Quirk GJ, Repa C, LeDoux JE. 1995. Fear conditioning enhances short-latency auditory responses of lateral amygdala neurons: Parallel recordings in the freely behaving rat. Neuron 15: 1029-1039.

Rescorla RA. 2001. Retraining of extinguished Pavlovian stimuli. J Exp Psychol Anim Behav Process 27: 115-124.

Rescorla RA. 2004. Spontaneous recovery. Learn Mem 11: 501-509.
Rescorla RA, Wagner AR. 1972. A theory of Pavlovian conditioning: Variations in the effectiveness of reinforcement and nonreinforcement. Appleton-Century-Crofts, New York.

Robbins SJ. 1990. Mechanisms underlying spontaneous recovery in autoshaping. J Exp Psychol Anim Behav Process 16: 235-249.

Ryu J, Futai K, Feliu M, Weinberg R, Sheng M. 2008. Constitutively active Rap2 transgenic mice display fewer dendritic spines, reduced extracellular signal-regulated kinase signaling, enhanced long-term depression, and impaired spatial learning and fear extinction. J Neurosci 28: $8178-8188$.

Schiller D, Levy I, Niv Y, LeDoux JE, Phelps EA. 2008. From fear to safety and back: Reversal of fear in the human brain. J Neurosci 28: 1151711525.

Sotres-Bayon F, Bush DE, LeDoux JE. 2007. Acquisition of fear extinction requires activation of NR2B-containing NMDA receptors in the lateral amygdala. Neuropsychopharmacology 32: 1929-1940.

Stough S, Shobe JL, Carew TJ. 2006. Intermediate-term processes in memory formation. Curr Opin Neurobiol 16: 672-678.

Suzuki A, Josselyn SA, Frankland PW, Masushige S, Silva AJ, Kida S. 2004. Memory reconsolidation and extinction have distinct temporal and biochemical signatures. J Neurosci 24: 4787-4795.

Urcelay GP, Wheeler DS, Miller RR. 2009. Spacing extinction trials alleviates renewal and spontaneous recovery. Learn Behav 37: 60-73.

Waltereit R, Dammermann B, Wulff P, Scafidi J, Staubli U, Kauselmann G, Bundman M, Kuhl D. 2001. Arg3.1/Arc mRNA induction by Ca2+ and cAMP requires protein kinase $\mathrm{A}$ and mitogen-activated protein kinase/extracellular regulated kinase activation. J Neurosci 21: 5484-5493.

Wisden W, Morris BJ. 1994. In situ hybridisation with synthetic oligonucleotide probes. In In situ hybridisation protocols for the brain (ed. W Wisden, BJ Morris), pp. 9-34. Academic Press, London, UK.

Woo NH, Teng HK, Siao CJ, Chiaruttini C, Pang PT, Milner TA, Hempstead BL, Lu B. 2005. Activation of p75NTR by proBDNF facilitates hippocampal long-term depression. Nat Neurosci 8: $1069-1077$.

Woods AM, Bouton ME. 2008. Immediate extinction causes a less durable loss of performance than delayed extinction following either fear or appetitive conditioning. Learn Mem 15: 909-920.

Yin Y, Edelman GM, Vanderklish PW. 2002. The brain-derived neurotrophic factor enhances synthesis of Arc in synaptoneurosomes. Proc Natl Acad Sci 99: 2368-2373.

Ying SW, Futter M, Rosenblum K, Webber MJ, Hunt SP, Bliss TV, Bramham CR. 2002. Brain-derived neurotrophic factor induces long-term potentiation in intact adult hippocampus: Requirement for ERK activation coupled to CREB and upregulation of Arc synthesis. $J$ Neurosci 22: 1532-1540.

Received May 19, 2010; accepted in revised form September 21, 2010. 


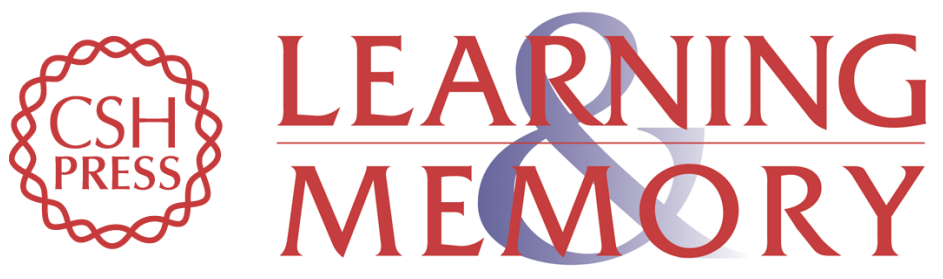

\section{The exclusive induction of extinction is gated by BDNF}

Anne Kirtley and Kerrie L. Thomas

Learn. Mem. 2010, 17:

Access the most recent version at doi:10.1101//m.1877010

Supplemental
Material http://learnmem.cshlp.org/content/suppl/2010/11/19/17.12.612.DC1

References This article cites 62 articles, 29 of which can be accessed free at: http://learnmem.cshlp.org/content/17/12/612.full.html\#ref-list-1

License

Email Alerting Receive free email alerts when new articles cite this article - sign up in the box at the Service top right corner of the article or click here. 